\title{
Voltage Flicker Parameter Estimation Based On Improved Analytic Energy Operator And Novel K-B Mutual Convolution Window
}

\author{
Yunfeng Li, Yunpeng Gao, Senior Member, IEEE, Yinghui Feng, Yijia Cao, Senior Member, \\ IEEE, and Yanqing Zhu
}

\begin{abstract}
It is difficult to measure voltage flicker parameter accurately and in real time under a complex power grid environment, a voltage flicker envelope extraction algorithm based on multi-point differential improved analytic energy operator (IAEO) is proposed, which simplified formula for extracting flicker envelope and a novel K-B optimal mutual convolution window function is constructed. Then, the correction formula of flicker amplitude and frequency is derived based on the three-spectral line interpolation of the novel K-B optimized mutual convolution window, and the estimation algorithm of voltage flicker parameter is proposed based on the IAEO and the novel K-B mutual convolution window. Finally, a voltage-flickerparameter-estimation platform based on virtual instrument is developed. The simulation and experimental results show that the proposed algorithm can effectively measure voltage flicker parameter under single frequency modulation, multi-frequency modulation and fundamental frequency fluctuation. In addition, it can effectively overcome harmonics, interharmonics and noise interference. Compared with the existing estimation algorithm, the flicker envelope extraction is simpler, the measurement result is more accurate, and it is easy to implement embedded.
\end{abstract}

Index Terms-Voltage flicker, analytic energy operator (AEO), window function, FFT, three-spectral line interpolation.

\section{INTRODUCTION}

$\mathrm{W}$ ith the development of new energy power generation technology and the increase of impact load, the problem of power grid voltage fluctuation and flicker becomes increasingly servere, which seriously affects the reliability of industrial production and the electricity needs in our daily lives[1-4]. Therefore, it is of great significance to accurate measurement and analysis of voltage flicker parameter for tracing and suppressing voltage flicker[5-7].

The square measurement method recommended by IEC is widely used due to its simple calculation and no attenuation coefficient. However, there is a frequency doubling component after square, which is not suitable for multifrequency flicker measurement[8]. A synchronous demodulation method is proposed to extract the flicker

This work was supported in part by the National Natural Science Foundation of China under Grant 51777061.

Y. Li, Y. Gao, F. Feng, Y. Cao and Y. Zhu are with the College of Electrical and Information Engineering, Hunan University, Changsha 410082, China (Corresponding author:Yunpeng Gao ,e-mail: gaoyp@hnu.edu.cn). envelope to avoid the influence of frequency doubling component on the estimation of flicker parameter[9]. But, it is difficult to measure accurately under high-frequency harmonic interference. In [10, 11], wavelet transform is used to measure flicker parameter, but it is difficult to select the wavelet generating function. In $[12,13]$, demodulation of flicker envelope component is achieved based on Hilbert transform. However, the measurement results are susceptible to noise interference. In $[14,15]$, the time-frequency matrix of flicker signal is obtained through S-transform, and then the flicker parameter estimation is realized. However, it is difficult to meet the real-time requirement of flicker parameter estimation, due to the large amount of calculation.

To realize the real-time and accurate measurement of flicker parameter, a flicker parameter estimation method based on energy operator is proposed in [16, 17], but energy operator demodulation has high frequency error and is sensitive to voltage mutation. Interval sampling is used to improve the energy operator for flicker envelope extraction[18], but highfrequency errors still exist. In [19], FFT spectral analysis method is used for rapid estimation of flicker parameter, but inherent spectrum leakage and fence effect restricted the measurement accuracy of flicker parameter, and the extraction accuracy of flicker envelope is affected by the change of frequency and amplitude.

In order to achieve rapid accurate measurement and analysis of flicker parameter under a complex power grid environment, an algorithm is developed to measure flicker parameter in real time and facilitate embedded implementation. In this paper, the AEO is improved based on multi-point differential, the flicker envelope extraction formula is simplified, and the voltage flicker envelope signal is extracted accurately. The function of Kaiser window and Blackman-Harris mutual convolution optimization window with narrow main lobe width and lower side lobe peak potential (novel K-B mutual convolution window for short) is constructed, and the threespectral line interpolation modified FFT formula based on the novel K-B mutual convolution window is derived. A voltage flicker parameter estimation algorithm based on IAEO and a novel K-B mutual convolution window is proposed. On these grounds, a voltage-flicker-parameter-estimation platform based on a virtual instrument is built, and the validity of the proposed algorithm is verified by simulation and experiment. 
The organization of this paper is as follows. Section II shows the voltage flicker model and flicker envelope extraction of the IAEO. Section III introduces the algorithm of flicker parameter estimation. Section IV analyses the simulation results. Section V introduces the experimental platform. Finally, Section VI concludes the paper.

\section{REAL-TIME EXTRACTION OF VOLTAGE FLICKER ENVELOPE}

\section{A. Voltage Flicker Model}

Voltage flicker can be regarded as the result of fundamental frequency voltage in power grid modulated by amplitude modulation of voltage fluctuation component[20], and mathematical model expressed as

$$
\begin{aligned}
u(t) & =a(t) \cos \left(\omega_{0} t+\theta_{0}\right) \\
& =U_{0}[1+v(t)] \cos \left(\omega_{0} t+\theta_{0}\right) \\
& =U_{0}\left[1+m_{i} \cos \left(\omega_{i} t+\theta_{i}\right)\right] \cos \left(\omega_{0} t+\theta_{0}\right)
\end{aligned}
$$

where $U_{0}$ is the voltage amplitude of the power grid, $\omega_{0}$ and $\theta_{0}$ are angular frequency and initial phase of the power grid power, $v(t)$ is the fluctuation component of voltage flicker signal, $m_{i}, w_{i}$ and $\theta_{i}$ are the amplitude modulation coefficient, amplitude modulation angle frequency and initial phase angle of voltage flicker fluctuation component amplitude modulation signal.

The discrete model of voltage flicker signal is obtained by discrete sampling according to equation (1)

$$
\begin{aligned}
R[u(n)] & =u(n)\left[\frac{-u_{j}(n+2)+8 u_{j}(n+1)-8 u_{j}(n-1)-u_{j}(n-2)}{12}\right] \\
& -u_{j}(n)\left[\frac{-u(n+2)+8 u(n+1)-8 u(n-1)-u(n-2)}{12}\right] \\
& =\left\{-u(n) * u_{j}(n+2)+8 u(n) * u_{j}(n+1)-8 u(n) * u_{j}(n-1)+u(n) * u_{j}(n-2)\right. \\
& \left.+u_{j}(n) * u(n+2)-8 u_{j}(n) * u(n+1)+8 u_{j}(n) * u(n-1)-u_{j}(n) * u(n-2)\right\} \div 12
\end{aligned}
$$

Substitute equation (2) into equation (4) to obtain

$$
\begin{aligned}
R[u(n)] & =\left\{-a(n) \cos \left(\Omega_{0} n+\theta_{0}\right) *\left[a(n+2) \sin \left(\Omega_{0}(n+2)+\theta_{0}\right)\right]+8 a(n) \cos \left(\Omega_{0} n+\theta_{0}\right) *\left[a(n+1) \sin \left(\Omega_{0}(n+1)+\theta_{0}\right)\right]\right. \\
& \left.-8 a(n) \cos \left(\Omega_{0} n+\theta_{0}\right) *\left[a(n-1) \sin \left(\Omega_{0}(n-1)+\theta_{0}\right)\right]+a(n) \cos \left(\Omega_{0} n+\theta_{0}\right) *\left[a(n-2) \sin \left(\Omega_{0}(n-2)+\theta_{0}\right)\right]\right\} \\
& +\left\{a(n) \sin \left(\Omega_{0} n+\theta_{0}\right) *\left[a(n+2) \cos \left(\Omega_{0}(n+2)+\theta_{0}\right)\right]-8 a(n) \sin \left(\Omega_{0} n+\theta_{0}\right) *\left[a(n+1) \cos \left(\Omega_{0}(n+1)+\theta_{0}\right)\right]\right. \\
& \left.+8 a(n) \sin \left(\Omega_{0} n+\theta_{0}\right) *\left[a(n-1) \cos \left(\Omega_{0}(n-1)+\theta_{0}\right)\right]-a(n) \sin \left(\Omega_{0} n+\theta_{0}\right) *\left[a(n-2) \cos \left(\Omega_{0}(n-2)+\theta_{0}\right)\right]\right\} \div 12 \\
& =\left\{-\sin 2 \Omega_{0}[a(n) a(n+2)+a(n) a(n-2)]+8 \sin \Omega_{0}[a(n) a(n+1)+a(n) a(n-1)]\right\} \div 12 \\
& \approx\left[a^{2}(n)\left(8 \sin \Omega_{0}-\sin 2 \Omega_{0}\right)\right] \div 6 \\
& =U_{0}{ }^{2}\left[1+\sum_{i=1}^{s} m_{i} \cos \left(\omega_{i} n+\theta_{i}\right)\right]^{2} *\left(8 \sin \Omega_{0}-\sin 2 \Omega_{0}\right) \div 6 \\
& =U_{0}^{2}\left[1+2 \sum_{i=1}^{s} m_{i} \cos \left(\omega_{i} n+\theta_{i}\right)+\sum_{i=1}^{s} m_{i}{ }^{2} \cos ^{2}\left(\omega_{i} n+\theta_{i}\right)\right. \\
& \left.+2 \sum_{i, j=1, i \neq j}^{s} m_{i} m_{j} \cos \left(\omega_{i} n+\theta_{i}\right) \cos \left(\omega_{j} n+\theta_{j}\right)\right]^{*}\left(8 \sin \Omega_{0}-\sin 2 \Omega_{0}\right) \div 6
\end{aligned}
$$

Since the amplitude modulation coefficient of flicker fluctuation component $m_{i}$ is usually far less than $10 \%$ of the power grid fundamental frequency voltage amplitude $U_{0}$, and the flicker frequency $f_{i}$ fluctuates among $[0.05,35] \mathrm{Hz}$, and the number of flicker fluctuation signal $s$ is small, equation (5) is simplified and the smaller part in equation (5) is ignored, only 
$C(n)$ is retained. Thus, the simplified extraction formula of voltage flicker envelope is expressed as

$$
v(n) \approx \frac{3 R[u(n)]}{U_{0}\left(8 \sin \Omega_{0}-\sin 2 \Omega_{0}\right)}-\frac{1}{2}
$$

The simplified formula of flicker envelope extraction does not need the square root operation, which reduces the amount of calculation. The speed of extracting flicker envelope of the IAEO is improved effectively, which is convenient for embedded implementation.

\section{FLICKER PARAMETER ESTIMATION ALGORITHM}

\section{A. Construction of A Novel K-B Mutual Convolution Window}

The window function with good performance can effectively reduce the spectrum leakage and picket-fence effect of FFT spectrum analysis error[23]. The traditional cosine window can not meet the performance requirements of low side lobe peak value and narrow main lobe at the same time, but Kaiser window can optimize the window function performance adaptively through parameter setting[24, 25]. A novel K-B mutual convolution window function is constructed by analyzing the spectral characteristics of the four-term Blackman-Harris cosine window and combining it with Kaiser window in this paper.

The time domain representation of the Kaiser window is

$$
w_{K}(n)=\frac{I_{0}\left(\beta \sqrt{1-\left(\frac{n}{N / 2}\right)^{2}}\right)}{I_{0}(\beta)}, \quad 0 \leq|n| \leq N / 2
$$

Where $I_{0}$ is the modified zero-order Bessel function, $\beta$ is the regulatory parameter of Kaiser window. Analyzing the relationship between the spectral response of Kaiser window and $\beta$ parameter, the Kaiser window $\beta=20$ is selected to construct a novel K-B mutual convolution window in this paper. Its spectral function is expressed as

$$
W_{K}(\omega)=\frac{N \sinh \left(\sqrt{\beta^{2}-(N \omega / 2)^{2}}\right)}{I_{0}(\beta) \sqrt{\beta^{2}-(N \omega / 2)^{2}}}
$$

The time domain representation of the unilateral 4-term Blackman-Harris cosine window is[26]

$$
w_{B}(n)=\sum_{l=0}^{L-1}(-1)^{l} b_{l} \cos (2 \pi n \cdot l / N)
$$

Where $n=1,2,3 \ldots N$ is the number of sampling points, $L=4$, The number $b_{i}$ satisfies $\sum_{l=0}^{L} b_{l}=1$ and $\sum_{l=0}^{L}(-1)^{l} b_{l}=0$ The coefficients are $b_{0}=0.35875, \quad b_{1}=0.48829$, $b_{2}=0.14128, b_{3}=0.01168$, which are expressed in the frequency domain as

$$
W_{B}(w)=\sum_{l=0}^{L-1}(-1)^{l} \frac{b_{l}}{2}\left[W_{R}\left(w-\frac{2 \pi}{N} l\right)+W_{R}\left(w+\frac{2 \pi}{N} l\right)\right]
$$

Where $W_{R}(w)=\frac{\sin (N w / 2)}{\sin (w / 2)} \mathrm{e}^{-\mathrm{j} w(N-1) / 2}$.

Through the time-domain convolution of Kaiser window and unilateral 4-term Blackman-Harris window, the discrete expression of the novel K-B mutual convolution window can be obtained

$$
w_{K-B}(n)=w_{K}(n) * w_{B}(n)=\sum_{i=0}^{n} w_{K}(i) \cdot w_{B}(n-i)
$$

According to the time-domain convolution theorem, timedomain convolution corresponds to the product in the frequency domain, so the frequency domain expression of the novel K-B mutual convolution window is

$$
W_{K-B}(\omega)=F T\left[w_{K}(n) * w_{B}(n)\right]=W_{K}(\omega) \cdot W_{B}(\omega)
$$

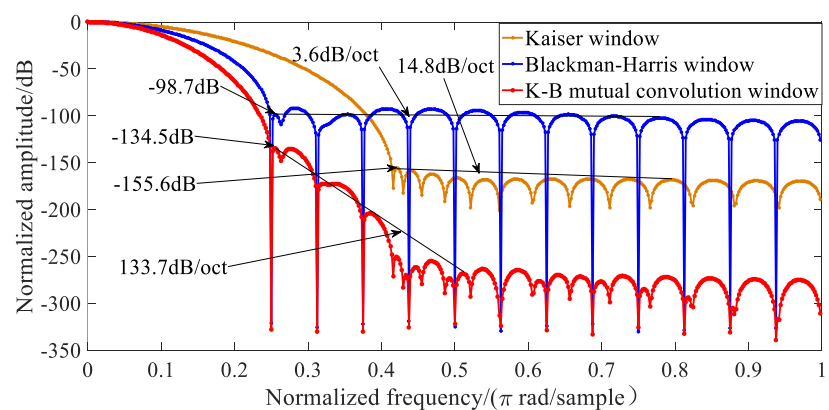

Fig.1 Normalized logarithmic spectrum of window functions

In this paper, Kaiser window function $\beta=20$, unilateral fourterm Blackman-Harris cosine window and the novel K-B optimized mutual convolution window constructed are taken as examples, normalized log-spectral analysis is carried out on them, and the analysis results are shown in Fig. 1. It can be seen from Fig 1 that the peak value of the side lobe of Kaiser window is $-155.6 \mathrm{db}$, the side lobe attenuation rate is $14.8 \mathrm{db} /$ oct, and OCT represents the octave. The peak value of the sidelobe of the Blackman-Harris cosine window function is $-98.7 \mathrm{~dB}$ and the attenuation rate of the sidelobe is $3.6 \mathrm{~dB} /$ OCT, while the peak value of the sidelobe of the K-B optimized mutual convolution window constructed in this paper is $-134.5 \mathrm{~dB}$ and the attenuation rate of the sidelobe is $133.7 \mathrm{~dB} / \mathrm{OCT}$. It can be seen that compared with the existing Blackman-Harris cosine window function and Kaiser window, the novel K-B optimized mutual convolution window function constructed in this paper has better sidelobe characteristics, narrower main lobe width, lower sidelobe peak value and faster sidelobe attenuation rate. Therefore, the novel K-B optimized mutual convolution window function has better performance.

\section{B. Three-spectral Line Interpolation Modified FFT}

A new mutual convolution window $w_{K-B}(n)$ is used to correct the flicker envelope component $v(n)$

$$
y(n)=v(n) w_{K-B}(n)
$$

The discrete FFT of $y(n)$ is obtained

$$
Y(k \Delta f)=\sum_{i=1}^{s} \frac{m_{i}}{2 j} e^{j \theta_{0}} W_{K-B}\left(2 \pi\left(k \Delta f-f_{0}\right) / f_{s}\right)
$$

Where $\Delta f=f_{s} / N$.

Due to the asynchronous sampling of FFT, the amplitude modulated signal frequency $k_{i} \Delta f$ cannot coincide with the sampling point ( $k_{i}$ is usually non-integer), which affects the accuracy of the flicker envelope analysis[27]. 
In this paper, FFT is improved by three-spectral line interpolation, and the corrected formula of amplitude and frequency of three-spectral line interpolation based on the novel K-B mutual convolution window is derived.

Assume that the maximum spectral line near the actual peak spectral line $k_{i}$ of the flicker envelope is $k_{j}$, and the left and right adjacent spectral lines of $k_{j}$ are $k_{j-1}$ and $k_{j+1}$ respectively[27, 28]. Let $\varepsilon=k_{i}-k_{j}(-0.5<\varepsilon<0.5)$, then the corresponding amplitudes of the three spectral lines are expressed as $x_{j+1}=\left|Y\left(k_{j+1} \Delta f\right)\right|, \quad x_{j}=\left|Y\left(k_{j} \Delta f\right)\right|, \quad x_{j-1}=\left|Y\left(k_{j-1} \Delta f\right)\right|$, Introducing parameter $\gamma$, and obtain the expression as

$$
\gamma=\frac{x_{j+1}-x_{j-1}}{x_{j}}
$$

Substituting the parameter $\varepsilon$ into equation (15), get

$$
\gamma=\frac{\left|W_{K-B}(1-\varepsilon)\right|-\left|W_{K-B}(-1-\varepsilon)\right|}{W_{K-B}(-\varepsilon) \mid}
$$

$\varepsilon$ is valued within $[-0.5,0.5]$, and the corresponding value of $\gamma$ can be obtained from equation (16), denoting $\gamma=h(\varepsilon)$. The polynomial fitting is performed by the polyfit function in MATLAB, and the parameter $\varepsilon$ and frequency expression in the three-spectral line interpolation correction formula based on the novel K-B mutual convolution window can be obtained as

$$
\begin{gathered}
\varepsilon=1.89128974 \gamma-0.12995728 \gamma^{3} \\
+0.01882393 \gamma^{5}-0.00335439 \gamma^{7} \\
f_{i}=\left(k_{j}+\varepsilon\right) \Delta f
\end{gathered}
$$

Similarly, by weighting the spectral line amplitude of $x_{j-1}, x_{j}$ and $x_{j+1}$, the correction formula of the flicker amplitude $m_{i}$ can be obtained as

$$
\begin{aligned}
m_{i}= & 2\left(x_{j-1}+2 x_{j}+x_{j+1}\right) /\left[\left|W_{K-B}\left(\frac{2 \pi(-\varepsilon-1)}{N}\right)\right|\right. \\
& \left.+2\left|W_{K-B}\left(\frac{2 \pi(-\varepsilon)}{N}\right)\right|+\left|W_{K-B}\left(\frac{2 \pi(-\varepsilon+1)}{N}\right)\right|\right] \\
= & \left(x_{j-1}+2 x_{j}+x_{j+1}\right) N^{-1} g(\varepsilon) \\
& g(\varepsilon)=2.40075915+0.31827223 \varepsilon^{2} \\
& +0.02184051 \varepsilon^{4}+0.00105347 \varepsilon^{6}
\end{aligned}
$$

\section{Voltage Flicker Parameter Estimation Procedures}

The voltage flicker parameter estimation procedure of the improved analytical energy operator and the novel K-B mutual convolution window proposed in this paper is shown in Fig. 2. The specific implementation steps are as follows:

(1) An improved analytical energy operator based on multipoint differential is used to obtain voltage flicker envelope signal.

(2) A novel K-B mutual convolution window is constructed.

(3) The envelope component is weighted with the novel K-B mutual convolution window.

(4) The discrete spectrum is obtained by FFT spectrum analysis of weighted sequences.

(5) Three maximum amplitude spectral lines are found through the peak-search procedure at $x_{j+1}, x_{j}$ and $x_{j-1}$.
(6) The parameters $\varepsilon$ and $g(\varepsilon)$ in frequency and amplitude modification formula is calculated by curve fitting.

(7) According to formulas (18) and (19), the frequency $f_{i}$ and amplitude $m_{i}$ modification formula of three-spectral line interpolation based on a novel K-B mutual convolution window are derived.

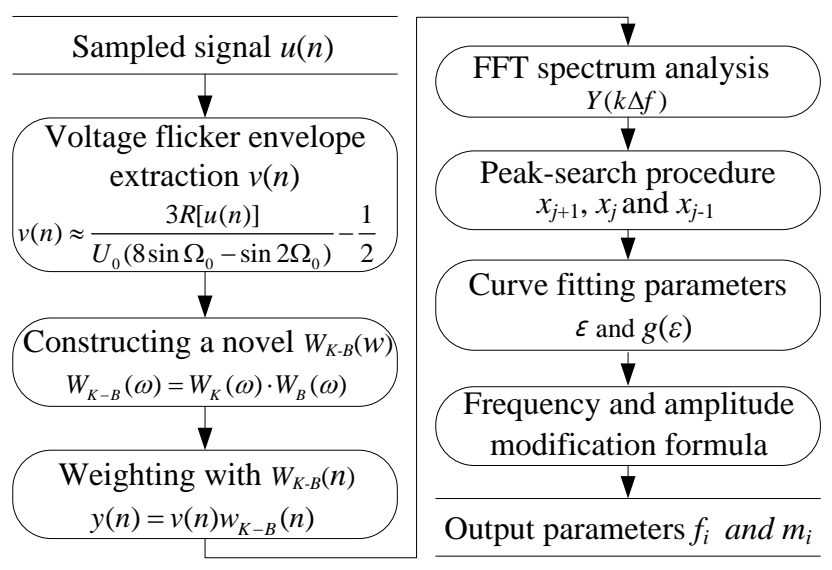

Fig. 2 Procedures of the IAEO for voltage flicker parameter estimation

\section{ANALYSIS OF SIMULATION RESULTS}

\section{A. Single Frequency Flicker Envelope Modulation}

To verify the effectiveness and accuracy of the voltage flicker parameter estimation method based on the improved analytic energy operator and novel K-B optimized mutual convolution window proposed in this paper.The flicker modulation signal with only a single frequency modulation component for simulation analysis in MATLAB. Assuming that the grid voltage amplitude $U_{0}=1 \mathrm{~V}$, the fundamental frequency $f_{0}$ is $50 \mathrm{~Hz}$, the amplitude modulation wave voltage frequency fluctuation range is $0.5 \sim 35 \mathrm{~Hz}$, and the amplitude modulation wave voltage fluctuation amplitude $m_{i}$ changes range $0 \sim 0.1$ p.u. (p.u. is a nominal value).

The relative error between amplitude and frequency of flicker envelope can be calculated by

$$
\left\{\begin{array}{l}
e_{m}=\frac{m_{c}-m_{i}}{m_{i}} \times 100 \% \\
e_{f}=\frac{f_{c}-f_{i}}{f_{i}} \times 100 \%
\end{array}\right.
$$

The amplitude relative error $e_{m}$ and frequency relative error $e_{f}$ of flicker envelope parameters are shown in Table 1, The logarithmic normalization of relative frequency errors is shown in Fig. 3.

As can be seen from Table I and Fig 3, when the amplitude modulation signal with only a single frequency component, the relative error of the flicker amplitude value is within $2 \%$, and the frequency error is within $10^{-6} \%$. Therefore, it can be seen that this method has high accuracy in the measurement of flicker envelope parameter, and meets the error requirements of IEC standard for flicker measurement. 


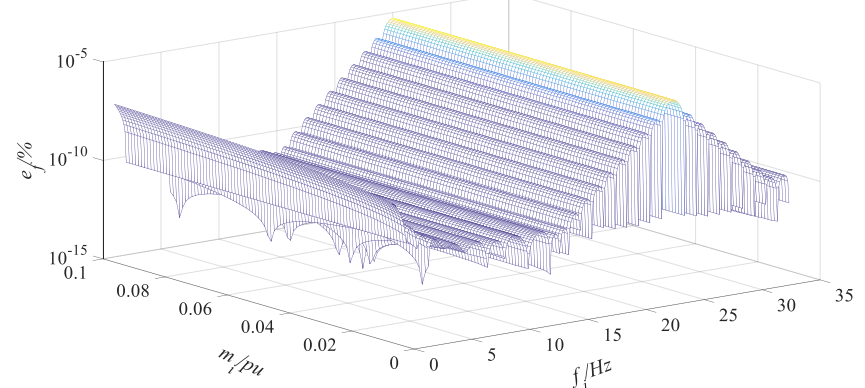

Fig. 3 Logarithmic normalization of relative frequency errors

TABLE I

VOLTAGE FLICKER MEASUREMENT ERROR OF SINGLE FREQUENCY

\begin{tabular}{cccc}
\hline $\begin{array}{c}\text { Modulation } \\
\text { frequency } \\
/ \mathrm{Hz}\end{array}$ & $\begin{array}{c}\text { Modulation } \\
\text { Magnitude } \\
/ \%\end{array}$ & $e_{m} / \%$ & $e_{f} / \%$ \\
\hline 1 & 0.25 & $-2.194 \mathrm{E}-3$ & $-1.352 \mathrm{E}-9$ \\
2 & 0.53 & $-8.783 \mathrm{E}-3$ & $5.445 \mathrm{E}-11$ \\
4 & 0.7 & $-3.519 \mathrm{E}-2$ & $1.981 \mathrm{E}-11$ \\
8 & 0.58 & $-1.416 \mathrm{E}-1$ & $1.583 \mathrm{E}-11$ \\
8.8 & 0.4 & $-1.716 \mathrm{E}-1$ & $-1.153 \mathrm{E}-11$ \\
15 & 0.16 & $-3.218 \mathrm{E}-1$ & $8.053 \mathrm{E}-12$ \\
24 & 0.1 & -1.355 & $-5.299 \mathrm{E}-12$ \\
\hline
\end{tabular}

\section{B. Multiple Frequency Flicker Envelope Modulation}

As the actual flicker envelope signal contains multiple modulation frequency components. To verify the accuracy of flicker parameter measurement under multi-frequency modulation of the proposed method, the voltage flicker envelope signal parameters and measurement results of multifrequency components are shown in Table II.

TABLE II

VOLTAGE FLICKER MEASUREMENT ERROR OF MULTIPLE FREQUENCIES

\begin{tabular}{cccc}
\hline $\begin{array}{c}\text { Modulation } \\
\text { Magnitude }\end{array}$ & $\begin{array}{c}\text { Modulation } \\
\text { frequency } \\
/ \mathrm{Hz}\end{array}$ & $e_{m} / \%$ & $e_{f} / \%$ \\
\hline 0.03 & 1 & 3.4974 & $5.2186 \mathrm{E}-7$ \\
0.07 & 2 & 0.3126 & $-3.3071 \mathrm{E}-7$ \\
0.1 & 8.8 & -0.1716 & $-8.1351 \mathrm{E}-10$ \\
\hline
\end{tabular}

It can be seen from Table II that the measurement accuracy of flicker parameters under multi-frequency modulation of the method in this paper still meets the error of IEC standard. Therefore, the algorithm proposed in this paper can realize the estimation of flicker parameter under multi-frequency modulation.

\section{Effects of Harmonic And Interharmonic Contamination}

TABLE III

VOLTAGE FLICKER MEASUREMENT ERROR OF MODULATION AMPLITUDE WITH HARMONIC CONTAMINATION

\begin{tabular}{cccccc}
\hline \multirow{2}{*}{ Case } & Condition & Harmonic frequency & Harmonic amplitude & $e_{m} / \%$ & $e_{f} / \%$ \\
\hline $\mathrm{a}$ & Flicker with no harmonic & - & 0 & -0.0551 & $1.7781 \mathrm{E}-11$ \\
$\mathrm{~b}$ & Flicker with $3^{\text {rd }}$ harmonic & 150 & 3.5 & -0.0793 & $19909 \mathrm{E}-11$ \\
$\mathrm{c}$ & Flicker with interharmonic & 186 & 2 & -0.0528 & $7.6708 \mathrm{E}-11$ \\
$\mathrm{~d}$ & Flicker with $5^{\text {rd }}$ harmonic and interharmonic & $250 ; 186$ & $5 ; 2$ & -0.0767 & $1.0611 \mathrm{E}-11$ \\
\hline
\end{tabular}

Since there are a large number of harmonics and interharmonics in the actual power grid, in order to analyze the influence of harmonics and interharmonics on the algorithm in this paper, voltage flicker signals of the integer harmonic and interharmonic are added respectively to measure flicker parameter. The set flicker frequency is $5 \mathrm{~Hz}$, amplitude modulation wave coefficient $m_{i}$ is 0.1 p.u., and voltage fundamental frequency $f_{0}$ is $50 \mathrm{~Hz}$. The harmonic and interharmonic parameters set are shown in Table III. The corresponding flicker frequency relative errors are shown in Fig. 4.

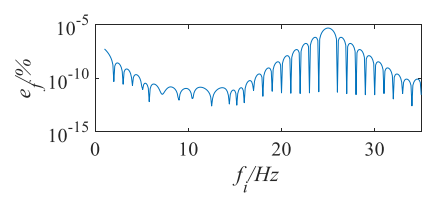

(a)With no hamonic

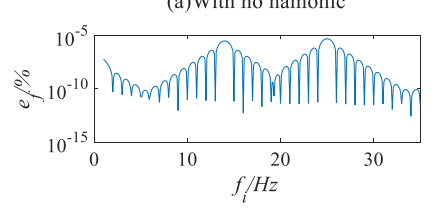

(c)With interhamonic

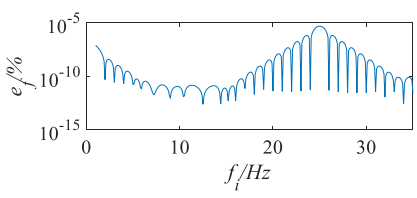

(b)With 3rd hamonic

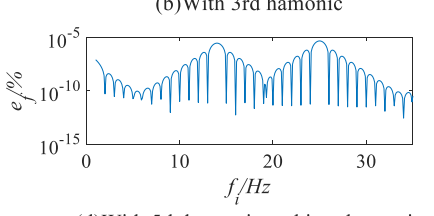

(d)With 5th hamonic and interhamonic
Fig. 4 The relative errors of flicker frequency with harmonic and interharmonic

Through the analysis of the amplitude error and frequency error results in Table III and Fig. 4, it can be seen that the algorithm proposed in this paper can effectively eliminate the influence of various harmonics on the estimation of flicker parameter.

\section{Effects of the Fluctuation of Fundamental Frequency}

There may be fundamental frequency fluctuation in actual power grid operation, which will cause further influence on spectrum leakage. Therefore, voltage flicker parameters are detected under the case of fundamental frequency fluctuation in this paper. The fluctuation range of the fundamental frequency $f_{0}$ is $49.5 \sim 50.5 \mathrm{~Hz}$, the frequency of the voltage flicker envelope signal fluctuates among $1 \sim 35 \mathrm{~Hz}$, and the amplitude modulation wave coefficient of the voltage flicker is $0.1 \mathrm{pu}$. The measurement errors of voltage flicker amplitude $m_{i}$ and frequency $f_{i}$ of the proposed algorithm are shown in Fig. 5 and Fig. 6 , respectively.

It can be seen from Fig. 5 and Fig. 6 that under the case of fundamental frequency fluctuation of power grid, the measurement errors of voltage flicker frequency are controlled below $10^{-6} \%$ as a whole. In addition, the relative error of the flicker modulation amplitude $m_{i}$ caused by the fundamental frequency change are less than $4 \%$. Therefore, the algorithm in this paper is not affected by fundamental frequency fluctuation and has strong anti-interference performance. 


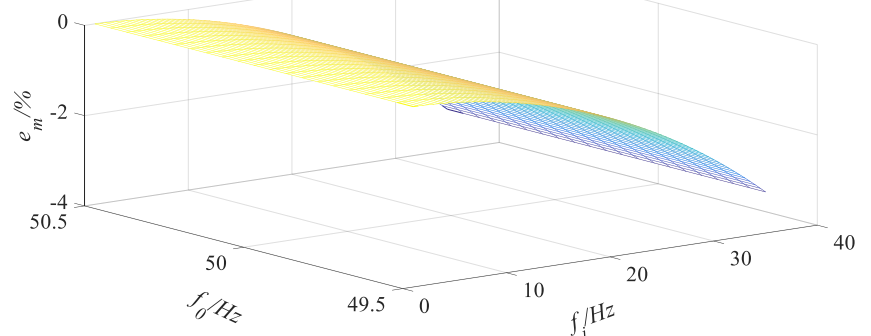

Fig. 5 Flicker amplitude measurement errors under fundamental frequency fluctuation

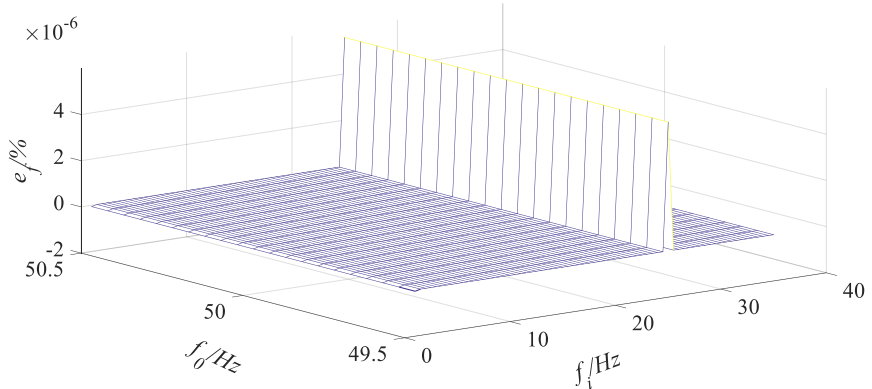

Fig. 6 Flicker frequency measurement errors under fundamental frequency fluctuation

\section{E. Effects of White Noise}

In order to verify the anti-noise performance of the proposed algorithm, white noise with SNR of $20 \mathrm{~dB} \sim 120 \mathrm{~dB}$ is added to the flicker signal, the voltage flicker amplitude is 0.1 p.u, and the frequency fluctuation among $1 \mathrm{~Hz} \sim 35 \mathrm{~Hz}$. The measurement results of voltage flicker parameters are shown in Fig. 7 and Fig. 8.

As shown in Fig. 7 and Fig. 8, even when the SNR of white noise is $20 \mathrm{~dB}$, the measurement errors of the flicker parameters are still less than $10 \%$. When the signal noise is greater than $40 \mathrm{~dB}$, the measurement errors of the $m_{i}$ is less than $5 \%$, which shows that the algorithm proposed in this paper has good performance under noisy environment.

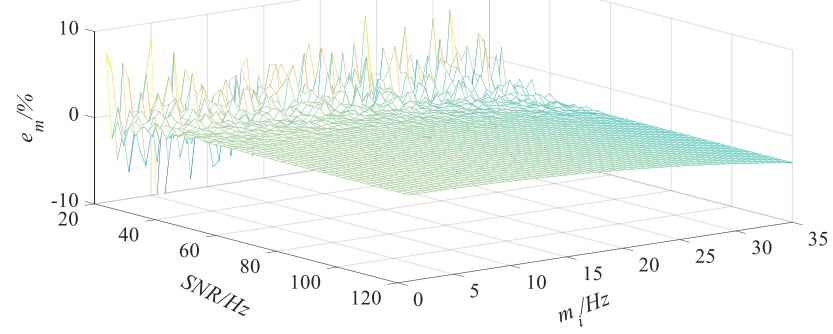

Fig.7 Measurement errors of flicker amplitude with noises

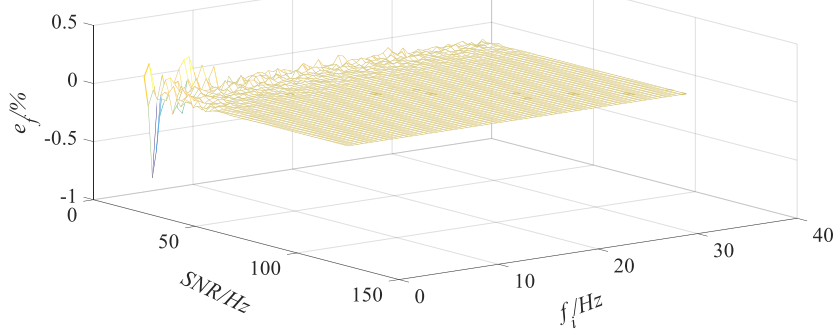

Fig. 8 Measurement errors of flicker frequency with noises

\section{F. Compared With Other parameter Estimation Algorithms}

To analyze the superiority of the proposed algorithm in flicker parameter estimation. The Teager energy operator, the improved Teager energy operator (ITEO), the square detection (SD) algorithm recommended by IEC flicker meter, the AEO and the algorithm proposed in this paper are selected to measure flicker parameter respectively. Flicker parameters are set according to the normalized flicker-meter response of the IEC 61000-4-15. The measurement results of flicker parameters are shown in Fig 9.

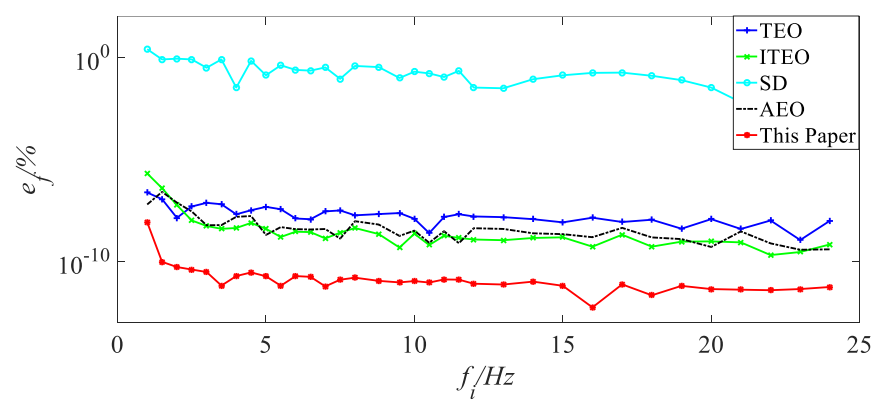

Fig. 9 Comparison of measurement results of different estimation algorithms

It can be seen from the results of flicker frequency error measurement shown in Fig. 9, the frequency errors of square detection flicker measurement are among $1 \mathrm{E}-2 \%$ and $1 \mathrm{E} 1 \%$, the frequency errors of TEO and ITEO are controlled below $1 \mathrm{E}-6 \%$, and the frequency errors of the proposed algorithm are controlled below $1 \mathrm{E}-10 \%$. The measurement errors of the proposed algorithm are much lower than the existing mainstream estimation methods under the full frequency band of flicker. In addition, the algorithm has the advantages of simple operation, small amount of calculation, high precision, and can be embedded.

\section{CONSTRUCTION AND VERIFICATION OF ESTIMATION PLATFORM}

To verify the practicability of the voltage flicker parameter estimation algorithm proposed in this paper, an experimental platform for flicker parameter estimation based on LabVIEW+PXI architecture is established. The experimental platform is shown in Fig 10.

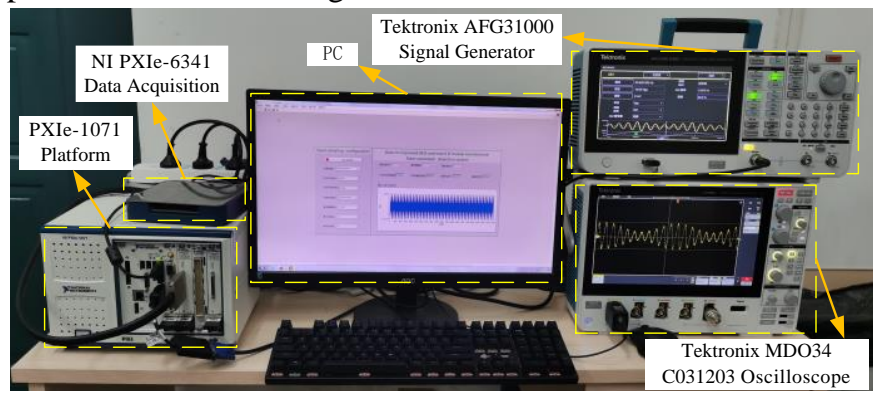

Fig. 10 The experiment platform

The voltage flicker model is set by selecting the voltage fluctuation parameters of the visual sensitivity $S=1$ given by the IEC standard, which are generated by MATLAB and imported into the Tektronix AFG31000 function generator. 
The measured voltage flicker waveform is monitored by an Tektronix MDO34 C031203 oscilloscope. The voltage flicker signal is sampled by NI PXIE-6341 data acquisition card, and then the collected discrete flicker signal is transmitted to the PXIE-1071 chassis through NI-DAQMX. The upper-computer software LabVIEW is programmed based on the algorithm proposed in this paper to realize flicker parameter estimation and calculation, real-time display of data waveform, data storage and printing, etc. The measured results are shown in Table IV.

TABLE IV

THE MEASUREMENT RESULTS OF FLICKER PARAMETER

\begin{tabular}{cccc}
\hline $\begin{array}{c}\text { Modulation } \\
\text { magnitude(\%.) }\end{array}$ & $\begin{array}{c}\text { Flicker } \\
\text { frequency }(\mathrm{Hz})\end{array}$ & $\begin{array}{c}\text { Relative errors } \\
\text { of } m_{i}(\%)\end{array}$ & $\begin{array}{c}\text { Relative errors } \\
\text { of } f_{i}(\%)\end{array}$ \\
\hline 0.882 & 2 & 0.187 & 0.076 \\
0.500 & 4 & 0.323 & 0.087 \\
0.250 & 8.8 & 0.279 & 0.063 \\
0.282 & 11 & 0.306 & 0.055 \\
0.348 & 13 & 0.203 & 0.081 \\
0.462 & 15 & 0.193 & 0.058 \\
0.640 & 19 & 0.237 & 0.082 \\
0.760 & 21 & 0.375 & 0.061 \\
\hline
\end{tabular}

As can be seen from Table IV that the measured flicker amplitude errors and flicker frequency errors of the voltage flicker parameter estimation algorithm based on the improved analytical energy operator and the novel K-B mutual convolution window proposed in this paper are controlled within $1 \%$. The error range meets the IEC Standard 61000-415 [20], so the algorithm proposed in this paper can effectively realize the accurate measurement of flicker parameter, which can be embedded.

\section{CONCLUSION}

A flicker parameter estimation algorithm based on improved analytic energy operator and novel K-B optimized mutual convolution window of the three-spectral line interpolation correction FFT is proposed in this paper. Simulation and experimental results show that the improved analytical energy operator can effectively improve the extraction accuracy of voltage flicker envelope, and the simplified formula for flicker envelope extraction is simple to implement and requires little calculation. The novel K-B mutual convolution window is characterized by lower side lobe peak value and narrower main lobe width. The threespectral line interpolation correction FFT algorithm based on the novel K-B mutual convolution window can effectively overcome the effect of spectral leakage and picket-fence. Based on this, the measurement results of the virtual instrument voltage flicker parameter estimation platform meet the standard of IEC61000-4-15. The proposed algorithm can effectively measure the voltage flicker envelope parameter in the case of single frequency modulation and multi-frequency modulation, and can overcome the interference of fundamental frequency fluctuation, harmonics and interharmonics. Compared with other estimation algorithms, the proposed algorithm has high measurement accuracy, strong real-time performance, and is easy to implement in embedded system, providing a new idea for voltage flicker parameter estimation and analysis under a complex power grid environment.

\section{REFERENCES}

[1] A. M. Alkandari, and S. A. Soliman, "Measurement of a power system nominal voltage, frequency and voltage flicker parameters," Int. J. Electr. Power Energy Syst., vol. 31, no. 7-8, pp. 295-301, 2009.

[2] C.-I. Chen, Y.-C. Chen, Y.-R. Chang, and Y.-D. Lee, "An Accurate Solution Procedure for Calculation of Voltage Flicker Components," IEEE Trans. Ind. Electron., vol. 61, no. 5, pp. 2370-2377, 2014.

[3] I. Sadinezhad, and V. G. Agelidis, "Frequency Adaptive Least-SquaresKalman Technique for Real-Time Voltage Envelope and Flicker Estimation," IEEE Trans. Ind. Electron., vol. 59, no. 8, pp. 3330-3341, 2012.

[4] A. Saadat, R.-A. Hooshmand, and M. Tadayon, "Flicker Propagation Pricing in Power Systems Using a New Short-Circuit-Based Method for Determining the Flicker Transfer Coefficient," IEEE Trans. Instrum. Meas., vol. 70, pp. 1-9, 2021.

[5] G. Wiczynski, and P. Kuwaek, "Voltage Distortion Influence on Flicker Severity Measurement by AMI Energy Meters," IEEE Trans. Ind. Electron., doi: 10.1109/TIE.2021.3120465.

[6] D. L. Geiger, and S. M. Halpin, "Assessing Voltage Fluctuations and Lamp Flicker Using RMS Voltages," IEEE Trans. Power Deliv., vol. 32, no. 6, pp. 2481-2488, 2017.

[7] L. Kukacka, P. Dupuis, M. Vik, A. Richter, and G. Zissis, "Confidence Intervals for Luminous Flicker Measurements: Comparison of Various Approaches," IEEE Trans. Ind. Appl., vol. 57, no. 5, pp. 5499-5506, 2021.

[8] X. Wu, M. Tian, Y. Zhu, Q. Zhao, and F. Liu, "A hybrid time and frequency domain digital implementation of flickermeter based on mirror image extension," IEEJ Trans. Electr. Electron. Eng., vol. 14, no. 7, pp. 982-989, 2019.

[9] P. Lidong, X. Xiangning, and X. Yonghai, "Measurement of Voltage Fluctuation and Flicker Based on Coherent Demodulation with Synchronous Digital Carrier," Int. Conf. Energy Environ. Technol., ICEET, pp. 168-171, 2009.

[10] S. J. Huang, and C. W. Lu, "Enhancement of Digital Equivalent Voltage Flicker Measurement via Continuous Wavelet Transform," IEEE Trans. Power Deliv., vol. 19, no. 2, pp. 663-670, 2004.

[11]S. A. Saleh, "Phaselet Transform Based Approach for Detecting Voltage Flickers Due to Distributed Generation Units," IEEE Trans. Ind. Appl., vol. 54, no. 5, pp. 5278-5292, 2018.

[12]H. Samet, M. Khosravi, T. Ghanbari, and M. Tajdinian, "A two-level neural network approach for flicker source location," Comput. Electr. Eng., vol. 92, 2021.

[13]N. C. F. Tse, J. Y. C. Chan, W.-H. Lau, and L. L. Lai, "Hybrid Wavelet and Hilbert Transform With Frequency-Shifting Decomposition for Power Quality Analysis," IEEE Trans. Instrum. Meas., vol. 61, no. 12, pp. 3225-3233, 2012.

[14]N. Eghtedarpour, E. Farjah, and A. Khayatian, "Effective Voltage Flicker Calculation Based on Multiresolution S-Transform," IEEE Trans. Power Deliv., vol. 27, no. 2, pp. 521-530, 2012.

[15]W. Yao, Q. Tang, Z. Teng, Y. Gao, and H. Wen, "Fast S-Transform for Time-Varying Voltage Flicker Analysis," IEEE Trans. Instrum. Meas., vol. 63, no. 1, pp. 72-79, 2014.

[16]T. K. Abdel-Galil, E. F. El-Saadany, and M. M. A. Salama, "Online Tracking of Voltage Flicker Utilizing Energy Operator and Hilbert Transform," IEEE Trans. Power Deliv., vol. 19, no. 2, pp. 861-867, 2004.

[17]M. A. Eldery, E. F. El-Saadany, and M. M. A. Salama, "A Simple Energy Operator Computational Method for Voltage Flicker Assessment," IEEE Trans. Power Deliv., vol. 21, no. 3, pp. 1743-1750, 2006.

[18]F. Li, Y. Gao, Y. Cao, and R. Iravani, "Improved Teager Energy Operator and Improved Chirp-Z Transform for Parameter Estimation of Voltage Flicker," IEEE Trans. Power Deliv., vol. 31, no. 1, pp. 245-253, 2016. 
[19] S. Sansheng, G. Liming, M. Lei, C. Zhuoya, and Z. Yuxiao, "Research on Flicker Measurement Algorithm Based on FFT," Energy Procedia, vol. 14, pp. 1709-1716, 2012.

[20]IEC61000-4-15, "Electromagnetic Compatibility (EMC) Part 4: Testing and Measurement Techniques Section 15: Flickermeter Functional and Design Specifications," Aug. 2010.

[21]H. Faghidi, and M. Liang, "Detection of Bearing Fault Detection From Heavily Contaminated Signals: A Higher-Order Analytic Energy Operator Method," J. Vib. Acoust.-Trans. ASME, vol. 137, no. 4, 2015.

[22]Y. Wang, L. Gu, L. Qing, X. Xu, and J. Shen, "An Improved k-Value Symmetrical Difference Analytic Energy Operator With HTFIF and LKCA for Bearing Fault Diagnosis," IEEE Access, vol. 9, pp. 3430734324, 2021.

[23]P. Yonglong, M. Xihao, L. Yabin, and F. Hailong, "A high-precision two-window three-spectral line interpolation harmonic detection algorithm," Chinese Control Conf., CCC, pp. 6595-6598, 2021.

[24]C. Liang, Z. Teng, J. Li, W. Yao, S. Hu, Y. Yang, and Q. He, “A Kaiser Window-Based S-transform for Time-Frequency Analysis of Power Quality Signals," IEEE Trans. Ind. Inform., pp. 1-1, 2021.

[25]N. Singh, and P. M. Pradhan, "Sharp Detection of Event's Onset in Seismic Signals With Asymmetrical Kaiser Window-Based STransform," IEEE Geosci. Remote Sens. Lett., vol. 16, no. 10, pp. 16201624,2019

[26]X. WANG, K. LEI, X. Yang, M. Li, and X. Wang, "Harmonic Analysis Based on Blackman-Harris Self-Multiplication Window," Proc. - Asia Conf. Power Electr. Eng., ACPEE, pp. 2165-2169, 2020.

[27]C. Wu, Y. Gao, C. Li, Y. Cao, and R. Iravani, "Analytic Mode Decomposition and Windowed Three-Point Interpolated Chirp-Z Transform for Voltage Flicker Components Detection," Electr. Power Syst. Res., vol. 186, 2020.

[28]L. Fan, and G. Qi, "Frequency estimator of sinusoid based on interpolation of three DFT spectral lines," Signal Processing, vol. 144, pp. 52-60, 2018.

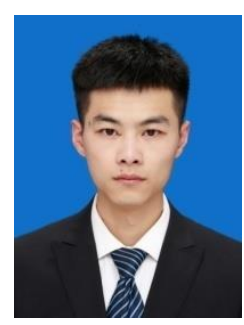

Yunfeng Li was born in Shaodong City, China in 1992. He is a Ph.D in Electronical Engineering at Hunan University, Changsha, China. He got the bachelor's degree in Electrical Engineering from China University of Mining and Technology, and a master's degree in Control Engineering from Hunan University,

$\mathrm{He}$ is researching Intelligent Information Processing, Power Quality Analysis, and Machine Learning. e-mail: yfli613@hnu.edu.cn

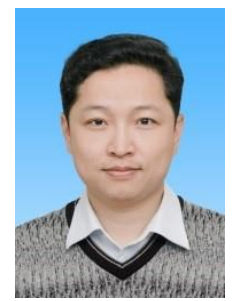

Yunpeng Gao (M'14-SM'18) was born in Liaoning China, in 1978. He received the B.Sc., M.Sc., and Ph.D. degrees in electrical engineering from Hunan University, Changsha, China, in 2001, 2004 and 2009 , respectively.

Currently, he is a Full Professor with the College of Electrical and Information Engineering, Hunan University. His interests include power distribution systems, power quality analysis, application of digital signal processing, and machine learning algorithms in power systems. e-mail: gaoyp@hnu.edu.cn

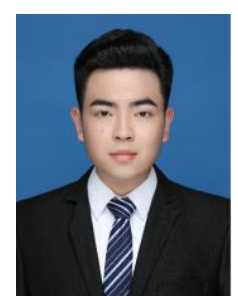

Yinghui Feng was born in Jiangxi Province, China, in 1997. He received his B.E. degree in measurement and control technology and instrumentation from Hunan University, Changsha,China, in 2020 . He is currently working towards the M.E. degree in Electronic and Information Engineering Hunan University, Changsha, China.

His research interests include Intelligent Information, modern signal process, and deep learning. e-mail: fengyh@hnu.edu.cn

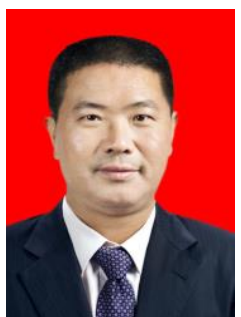

Yijia Cao (M'98-SM'13) received the B.E. degree from Xi'an Jiaotong University, Xian, China, in 1998, and the Master's and Ph.D. degree from Huazhong University of Science and Technology, Wuhan, China, in 1990 and 1994, respectively, all in electrical engineering. Currently, he is a Professor and the Vice President with Hunan University, Changsha, China.

His research interests include power system cascading failure, smart grid information technology, smart grid operation and optimization. E-mail: yijiacao@zju.edu.cn

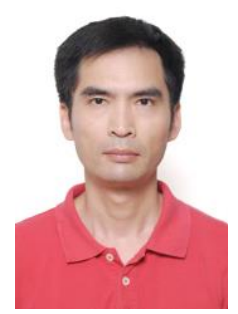

Yanqing Zhu was born in, Hunan, China, in 1978. He received the B.Sc., M.Sc., and Ph.D. degrees in electrical engineering from Hunan University, Changsha, China, in 2001, 2004, and 2008, respectively.

Currently, he is a Associate Professor with the College of Electrical and Information Engineering, Hunan University. His interests include power quality analysis, electric network testing and fault diagnosis, new energy technology, artificial intelligence.e-mail: zyq@hnu.edu.cn 